\title{
On the Uplink Achievable Rate of Massive MIMO System With Low-Resolution ADC and RF Impairments
}

\author{
Liangyuan Xu, Xintong Lu, Shi Jin, Senior Member, IEEE, Feifei Gao, Senior Member, IEEE, and Yongxu Zhu
}

\begin{abstract}
This paper considers channel estimation and uplink achievable rate of the coarsely quantized massive multiple-input multiple-output (MIMO) system with radio frequency (RF) impairments. We utilize additive quantization noise model (AQNM) and extended error vector magnitude (EEVM) model to analyze the impacts of low-resolution analog-to-digital converters (ADCs) and RF impairments respectively. We show that hardware impairments cause a nonzero floor on the channel estimation error, which contraries to the conventional case with ideal hardware. The maximal-ratio combining (MRC) technique is then used at the receiver, and an approximate tractable expression for the uplink achievable rate is derived. The simulation results illustrate the appreciable compensations between ADCs' resolution and RF impairments. The proposed studies support the feasibility of equipping economical coarse ADCs and economical imperfect RF components in practical massive MIMO systems.
\end{abstract}

Index Terms-Quantized massive MIMO, uplink rate, channel estimation, RF impairments, low-resolution ADC, MRC.

\section{INTRODUCTION}

M ASSIVE multi-input multi-output (MIMO), a promising technology for $5 \mathrm{G}$ mobile network, deploys a large number of radio frequency (RF) chains and analog-to-digital converters (ADCs) at the base station (BS) [1]. As the number and quality of ADCs and RF chains increase, the financial costs and energy dissipation will grow significantly, which motivates studies of equipping economical coarse ADCs and imperfect RF chains in massive MIMO system.

Under the assumption of additive quantization noise model (AQNM), the impacts of low-resolution ADCs on the uplink achievable rate of massive MIMO system were investigated in [2], [3], and the asymptotic downlink achievable rate was derived in [4]. For the special case of 1-bit quantization,

Manuscript received September 20, 2018; revised December 29, 2018 and January 19, 2019; accepted January 22, 2019. The associate editor coordinating the review of this paper and approving it for publication was Dr Christos Masouros. This work was supported in part by the National Natural Science Foundation of China under Grant $\{61831013,61771274,61531011\}$, and by Beijing Municipal Natural Science Foundation under Grant $\{4182030$, L182042\}. The work of S. Jin was supported in part by the National Science Foundation for Distinguished Young Scholars of China with Grant 61625106. (Corresponding author: Feifei Gao.)

L. Xu and F. Gao are with the Department of Automation, Tsinghua University, Beijing 100084, China (e-mail: xly18@mails.tsinghua.edu.cn; feifeigao@ieee.org)

$\mathrm{X}$. $\mathrm{Lu}$ and $\mathrm{S}$. Jin are with the National Mobile Communications Research Laboratory, Southeast University, Nanjing 210096, China (e-mail: luxintong@seu.edu.cn; jinshi@seu.edu.cn).

Y. Zhu is with the Wolfson School of Mechanical, Electrical and Manufacturing Engineering, Loughborough University, Leicestershire, LE11 3TU, UK (e-mail: yongxu.zhu.13@ucl.ac.uk). channel estimation and performance of massive MIMO system have been investigated in [5]. These studies, however, ignored RF impairments, e.g., amplifier nonlinearities, I/Q imbalance and phase noise.

On the other hand, the effects of I/Q imbalance were analyzed in [6]. To capture the aggregate impact of different types of RF impairments, [7] proposed a generalised error model, named extended EVM (EEVM). However, lowresolution ADCs were not taken into account.

The impacts of both ADCs and RF impairments on the energy efficiency, capacity and estimation were investigated in [8]. However, the overall impacts were modeled as additive Gaussian noise which is excessively general.

In this paper, we investigate the uplink achievable rate and channel estimation of massive MIMO system with both lowresolution ADCs and RF impairments. Instead of modeling these impacts as simple additive Gaussian noise, we utilize AQNM and EEVM model to capture the impacts of coarse ADCs and RF impairments respectively. Specifically, we first propose an approach for channel estimation under minimum mean square error (MMSE) criterion, and we demonstrate that the estimation accuracy is limited by both coarse ADCs and hardware impairments. Then, the maximal-ratio combining (MRC) technique is applied at the receiver with imperfect channel state information (CSI), and a tightly approximated tractable expression of the uplink achievable rate is derived. We show that increasing the number of receiver antennas could mitigate the performance degradation caused by both coarse ADCs and RF impairments. In addition, the appreciable compensations between ADCs resolution and RF impairments are illustrated, which indicates that the performance loss caused by severe RF impairments could be compensated by improving the resolution of ADCs, and vice versa. These compensations is valuable and could be used to optimize the financial costs and energy dissipation of massive MIMO system.

\section{System Model}

Consider a multi-user massive MIMO system consisted of a BS with $M$ antennas and $K$ single-antenna users, as demonstrated in Fig. 1. Assume that RF chains and ADCs of the $\mathrm{BS}$ are ideal. The received signal vector at the $\mathrm{BS}$ is

$$
\mathbf{y}=\sqrt{\rho} \mathbf{G} \mathbf{x}+\mathbf{n},
$$

where $\mathbf{G}$ is the $M \times K$ channel matrix with the $(m, k)$ th element $g_{m k}, \mathbf{x}$ denotes the $K \times 1$ symbols vector transmitted by $K$ 

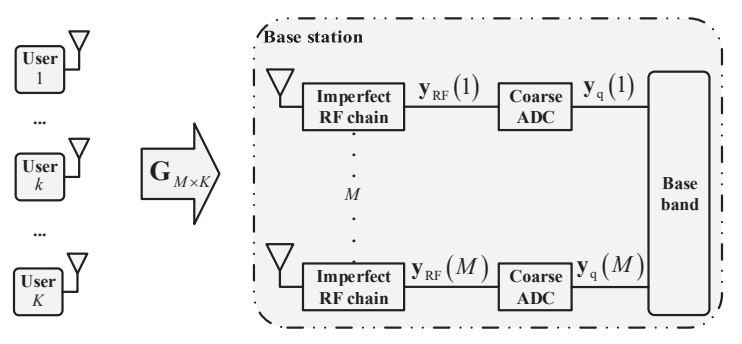

Fig. 1. Block diagram of multi-user massive MIMO system

TABLE I

$\mu$ For Different Quantization Bits $b$

\begin{tabular}{|c|c|c|c|c|c|}
\hline$b$ & 1 & 2 & 3 & 4 & 5 \\
\hline$\mu$ & 0.3634 & 0.1175 & 0.03454 & 0.009497 & 0.002499 \\
\hline
\end{tabular}

users, $\rho$ is the normalized average power of each user, and $\mathbf{n} \sim \operatorname{CN}(0, \mathbf{I})$ is the additive white Gaussian noise vector.

The channel coefficient between the $k$ th user and the $m$ th antenna of the BS is modeled as

$$
g_{m k}=h_{m k} \sqrt{\beta_{k}},
$$

where $h_{m k} \sim \mathcal{C N}(0,1)$ is the fast-fading coefficient, and $\beta_{k}$ presents both geometric attenuation and shadow fading of the $k$ th user to the whole antenna array [1].

With the existence of errors caused by imperfect RF chains, we should adopt EEVM to rewrite the received signal as [7, Chapter 7]

$$
\mathbf{y}_{\mathrm{RF}}=\sqrt{\rho} \chi \mathbf{G} \mathbf{x}+\mathbf{n}_{\mathrm{RF}}+\mathbf{n},
$$

where $\mathbf{y}_{\mathrm{RF}}$ is the received vector after imperfect RF chains, $\chi=\operatorname{diag}\{\chi(1), \cdots, \chi(M)\}, \mathbf{n}_{\mathrm{RF}}=\left\{n_{\mathrm{RF}}(1), \cdots, n_{\mathrm{RF}}(M)\right\}^{T}$, $n_{\mathrm{RF}}(m)$ denotes the additive distortion noise of the $m$ th $\mathrm{RF}$ chains, and $\chi(m)=\kappa(m) e^{J \varphi(m)}$ presents scaling and phase shift effects of the $m$ th RF chains with $|\kappa(m)| \leq 1$. The mapping of these parameters to particular type of RF impairment (e.g., nonlinearity, I/Q imbalance and phase noise) could be found in [7, Chapter 7]. For ease of derivation, we assume that $n_{\mathrm{RF}}(m)$ is Gaussian with $n_{\mathrm{RF}}(m) \sim \mathcal{C N}\left(0, \sigma_{m}^{2}\right)$, and impairments of all $\mathrm{RF}$ chains are in the same level with $\chi(m)=\chi$ and $\sigma_{m}=\sigma$ in the remainder of this paper.

Assuming the automatic gain control (AGC) is ideal and set properly, we can use AQNM to model the coarsely quantized outputs as [2]

$$
\mathbf{y}_{\mathrm{q}}=\eta \mathbf{y}_{\mathrm{RF}}+\mathbf{n}_{\mathrm{q}}=\eta \sqrt{\rho} \chi \mathbf{G} \mathbf{x}+\eta \mathbf{n}_{\mathrm{RF}}+\eta \mathbf{n}+\mathbf{n}_{\mathrm{q}},
$$

where $\mathbf{n}_{\mathrm{q}}$ is the additive quantization noise vector such that $\mathbf{n}_{\mathrm{q}}$ and $\mathbf{y}_{\mathrm{RF}}$ are uncorrelated, $\eta=1-\mu$, and $\mu$ is the inverse of signal-to-quantization-noise ratio. We define $\mathbf{P} \triangleq \chi \mathbf{G}$ as effective channel. Let $b$ denotes the quantization bits. Then, $\mu$ can be approximately expressed as $\mu=\frac{\pi \sqrt{3}}{2} 2^{-2 b}$ for $b>5$, and the values of $\mu$ for $b \leq 5$ are listed in Table I [9].

For given channel realizations $\mathbf{G}$, the covariance matrix of $\mathbf{n}_{\mathrm{q}}$ can be expressed as [9]

$$
\mathbf{C}_{\mathbf{n}_{\mathrm{q}}}=\mathbb{E}\left\{\mathbf{n}_{\mathrm{q}} \mathbf{n}_{\mathrm{q}}^{H} \mid \mathbf{G}\right\}=\mathbb{E}\left\{\eta(1-\eta) \operatorname{diag}\left\{\mathbf{y}_{\mathrm{RF}} \mathbf{y}_{\mathrm{RF}}^{H}\right\}\right\} .
$$

Assume that $\mathbf{C}_{x}$ is the covariance matrix of input signal with $\mathbf{C}_{x}=\mathbf{I}$, and $\mathbf{C}_{\mathbf{n}_{\mathrm{RF}}}$ is the covariance matrix of $\mathbf{n}_{\mathrm{RF}}$ with
$\mathbf{C}_{\mathbf{n}_{\mathrm{RF}}}=\sigma^{2} \mathbf{I}$ where $\sigma^{2}$ is variance of $n_{\mathrm{RF}}(m)$. Then, (5) can be simplified as

$$
\mathbf{C}_{\mathbf{n}_{\mathrm{q}}}=\eta(1-\eta) \operatorname{diag}\left\{\rho \chi \mathbf{G G}^{H} \chi^{H}+\mathbf{I}+\mathbf{C}_{\mathbf{n}_{\mathrm{RF}}}\right\} .
$$

\section{Channel Estimation}

We consider a block fading scenario where the channel remains constant during the coherent interval. Each interval is divided into two parts: one part for pilot sequences and the other for data. During pilot sequences transmission, each user transmits $\tau$ pilot symbols simultaneously. Combining $\tau$ quantized vectors of (4) into a matrix yields

$$
\mathbf{Z}_{\mathrm{q}}=\left[\mathbf{y}_{\mathrm{q}}^{1}, \cdots, \mathbf{y}_{\mathrm{q}}^{\tau}\right]=\eta \sqrt{\rho_{\mathrm{p}}} \mathbf{P} \boldsymbol{\Phi}^{T}+\eta \mathbf{N}_{\mathrm{RF}}+\eta \mathbf{N}+\mathbf{N}_{\mathrm{q}},
$$

where $\mathbf{Z}_{\mathrm{q}} \in \mathbb{C}^{M \times \tau}$ is the quantized outputs, $\rho_{\mathrm{p}}$ is the power of pilot sequences, $\mathbf{N}_{\mathrm{q}}, \mathbf{N}$ and $\mathbf{N}_{\mathrm{RF}}$ are matrix forms of $\mathbf{n}_{\mathrm{q}}$, $\mathbf{n}$ and $\mathbf{n}_{\mathrm{RF}}$ respectively, and $\boldsymbol{\Phi} \in \mathbb{C}^{\tau \times K}(\tau \geq K)$ denotes the pilot matrix. We take $\boldsymbol{\Phi}$ as $K$ columns of the $\tau \times \tau$ DFT (Discrete Fourier Transform) matrix such that $\boldsymbol{\Phi}$ is columnwise orthogonal.

Let us vectorize $\mathbf{Z}_{\mathrm{q}}$ and obtain

$$
\mathbf{z}_{\mathrm{q}}=\operatorname{vec}\left(\mathbf{Z}_{\mathrm{q}}\right)=\eta \sqrt{\rho_{\mathrm{p}}} \overline{\boldsymbol{\Phi}} \underline{\mathbf{p}}+\eta \underline{\mathbf{n}}_{\mathrm{RF}}+\eta \underline{\mathbf{n}}+\underline{\mathbf{n}}_{\mathrm{q}},
$$

where $\overline{\mathbf{\Phi}}=\left(\boldsymbol{\Phi} \otimes \mathbf{I}_{M}\right), \otimes$ denotes the Kronecker product, $\underline{\mathbf{p}}=$ $\operatorname{vec}(\mathbf{P})$ is the vector form of the effective channel $\mathbf{P}, \underline{\mathbf{n}}_{\mathrm{RF}}=$ $\operatorname{vec}\left(\mathbf{N}_{\mathrm{RF}}\right), \underline{\mathbf{n}}=\operatorname{vec}(\mathbf{N})$ and $\underline{\mathbf{n}}_{\mathrm{q}}=\operatorname{vec}\left(\mathbf{N}_{\mathrm{q}}\right)$.

Theorem 1. The linear minimum mean square error (LMMSE) estimator of $\mathbf{p}$ is [10]

$$
\underline{\widehat{\mathbf{p}}}=\mathbf{C}_{\underline{\mathbf{p}} \mathbf{z}_{\mathrm{q}}} \mathbf{C}_{\mathbf{z}_{\mathrm{q}}}^{-1} \mathbf{z}_{\mathrm{q}},
$$

where $\mathbf{C}_{\mathbf{p z}_{\mathrm{q}}}$ is the covariance matrix between $\underline{\mathbf{p}}$ and $\mathbf{z}_{\mathrm{q}}, \mathbf{C}_{\mathbf{z}_{\mathrm{q}}}$ is the covariance matrix of $\mathbf{z}_{\mathrm{q}}$, and $\widehat{\mathbf{p}}=\operatorname{vec}(\widehat{\mathbf{P}}) \overline{i s}$ the estimator of the effective channel. The normalized MSE is

$$
\mathrm{MSE}=\frac{\mathbb{E}\left\{\|\widehat{\mathbf{p}}-\underline{\mathbf{p}}\|_{2}^{2}\right\}}{M K}=\frac{\sum_{k=1}^{K}\left(\beta_{k}|\chi|^{2}-\alpha_{k} \beta_{k}|\chi|^{2}\right)}{K},
$$

where

$$
\alpha_{k} \triangleq \frac{\eta \rho_{\mathrm{p}} \tau|\chi|^{2} \beta_{k}}{\eta \rho_{\mathrm{p}} \tau|\chi|^{2} \beta_{k}+(1-\eta) \rho_{\mathrm{p}}|\chi|^{2} \sum_{k=1}^{K} \beta_{k}+\sigma^{2}+1} .
$$

Proof: Due to space limitations, derivations are omitted and can be found in a longer version of this paper [11].

Note that $\alpha_{k}$ is interpreted as the accuracy of the estimator and is characterized by the level of hardware impairments, pilot power and pilot length. Since the denominator of (11) is greater than the numerator, we have $0 \leq \alpha_{k} \leq 1$. When $\alpha_{k}=1$, MSE in (10) becomes zero, which means perfect CSI without estimation error. On the other hand, $\alpha_{k}=0$ means the worst estimator.

Remark 1. In the high SNR regime, if $\tau=K$, we have

$$
\lim _{\rho_{\mathrm{p}} \rightarrow \infty} \alpha_{k}=\frac{\eta K \beta_{k}}{\eta K \beta_{k}+(1-\eta) \sum_{i}^{K} \beta_{i}}<1,
$$




$$
\lim _{\rho_{\mathrm{p}} \rightarrow \infty} \mathrm{MSE}=\sum_{k=1}^{K}\left(\left(\frac{1}{K}-\frac{\eta \beta_{k}}{\eta K \beta_{k}+(1-\eta) \sum_{i}^{K} \beta_{i}}\right) \beta_{k}|\chi|^{2}\right) .
$$

Remark 1 indicates that there is a nonzero error floor as $\rho_{\mathrm{p}} \rightarrow \infty$ which contraries to the ideal hardware case. This nonzero error floor is characterized by the level of hardware impairments and cannot be eliminated by increasing SNR.

\section{Uplink Achievable Rate}

By using MRC technique with imperfect CSI obtained from (9), we can modify the quantized signal vector of (4) into

$$
\mathbf{r}=\widehat{\mathbf{P}}^{H} \mathbf{y}_{\mathrm{q}} .
$$

Substituting (4) into (14), we obtain

$$
\mathbf{r}=\eta \sqrt{\rho_{\mathrm{u}}} \widehat{\mathbf{P}}^{H} \mathbf{P x}+\eta \widehat{\mathbf{P}}^{H}\left(\mathbf{n}_{\mathrm{RF}}+\mathbf{n}\right)+\widehat{\mathbf{P}}^{H} \mathbf{n}_{\mathrm{q}} .
$$

The $n$th element of $\mathbf{r}$ can be expressed as

$$
r_{n}=\eta \sqrt{\rho_{\mathrm{u}}} \widehat{\mathbf{p}}_{n}^{H} \mathbf{p}_{n} x_{n}+\underbrace{\eta \widehat{\mathbf{p}}_{n}^{H}\left(\mathbf{n}_{\mathrm{RF}}+\mathbf{n}\right)+\widehat{\mathbf{p}}_{n}^{H} \mathbf{n}_{\mathrm{q}}+\eta \sqrt{\rho_{\mathrm{u}}} \sum_{k=1, k \neq n}^{K} \widehat{\mathbf{p}}_{n}^{H} \mathbf{p}_{k} x_{k},}_{\triangleq}
$$

where $\mathbf{p}_{n}$ is the $n$th column of $\mathbf{P}, \widehat{\mathbf{p}}_{n}$ is the $n$th column of $\widehat{\mathbf{P}}$, and the random variable $\xi$ presents noise-plus-interference with zero mean and variance

$\mathcal{I}_{\mathbf{G}}=\eta^{2} \widehat{\mathbf{p}}_{n}^{H} \mathbf{C}_{\mathbf{n}_{\mathrm{RF}}} \widehat{\mathbf{p}}_{n}+\eta^{2}\left\|\widehat{\mathbf{p}}_{n}\right\|_{2}^{2}+\widehat{\mathbf{p}}_{n}^{H} \mathbf{C}_{\mathbf{n}_{\mathrm{q}}} \widehat{\mathbf{p}}_{n}+\eta^{2} \rho_{\mathrm{u}} \sum_{\substack{k=1 \\ k \neq n}}^{K}\left|\widehat{\mathbf{p}}_{n}^{H} \mathbf{p}_{k}\right|^{2}$.

We model $\xi$ as additive Gaussian noise which is uncorrelated with $x_{n}$. Then, we can derive the ergodic uplink achievable rate of the $n$th user as

$$
R_{n}=\mathbb{E}\left\{\log _{2}\left(1+\frac{\rho_{\mathrm{u}} \eta^{2}\left|\widehat{\mathbf{p}}_{n}^{H} \mathbf{p}_{n}\right|^{2}}{\mathcal{I}_{\mathbf{G}}}\right)\right\}
$$

where the expectation is taken with respect to $h_{m n}$. Since we cannot directly derive a tractable expression from (17), an approximate expression is presented as follows

Theorem 2. The ergodic uplink achievable rate $R_{n}$ of the $\mathrm{n}$ th user can be approximated as

$$
\widetilde{R}_{n}=\log _{2}\left(1+\frac{\eta \rho_{\mathrm{u}}|\chi|^{2} \beta_{n}\left(\alpha_{n} M+1\right)}{\mathcal{D}}\right)
$$

where $\mathcal{D}$ is given by

$$
\mathcal{D}=\rho_{\mathrm{u}}|\chi|^{2}\left(\sum_{i}^{K}\left(\beta_{i}\right)-\eta \beta_{n}\right)+(1-\eta) \alpha_{n} \rho_{\mathrm{u}}|\chi|^{2} \beta_{n}+\sigma^{2}+1
$$

Proof: Due to space limitations, derivations are omitted and can be found in a longer version of this paper [11].

Theorem 2 shows the impacts of coarse ADCs, RF impairments and channel estimation errors on the achievable rate. Compared to the related works in [2], [5], [8], we consider more general case with both coarse ADC and RF impairments included. Since the expression in Theorem 2 is complicated, the compensations between ADCs resolution and
RF impairments are implicit. Compensations mean that the performance loss caused by severe RF impairments could be compensated by improving the resolution of ADCs, and vice versa. To gain insights into the compensations, we will investigate the following special cases of Theorem 2.

Remark 2. Assuming perfect CSI $\left(\alpha_{k}=1\right)$, the upper bound of $\widetilde{R}_{n}$ is

$$
\widetilde{R}_{n, \text { upper }}=\log _{2}\left(1+\frac{\beta_{n}+M \beta_{n}}{\frac{1}{\eta} \sum_{i=1}^{K}\left(\beta_{i}\right)+\left(\frac{1}{\eta}-2\right) \beta_{n}+\frac{1+\sigma^{2}}{\eta \rho_{\mathrm{u}}|\chi|^{2}}}\right) \text {. }
$$

If RF components are ideal and only low-resolution ADCs are considered, e.g., $|\chi|=1$ and $\sigma^{2}=0,(19)$ is consistent with the result in [2]. Note that $\eta, \sigma^{2}, \chi$ and $p_{u}$ merely appear in the denominator of (19), and $M$ only appears in the numerator. Therefore, it is easy to figure out that the loss of the uplink achievable rate caused by hardware impairments could always be compensated by increasing the number of antennas $M$. The compensation by increasing $p_{u}$, however, is unsatisfying since $p_{u}$ merely appears in the term $\left(1+\sigma^{2}\right) / \eta p_{u}|\chi|^{2}$. As $p_{u} \rightarrow \infty,\left(1+\sigma^{2}\right) / \eta p_{u}|\chi|^{2}$ will converge to zero and (19) will converge as well. The reason is that interferences among users deteriorate as $p_{u}$ increases.

Remark 3. The approximated achievable rate in (18) can be simplified to

$$
\widetilde{R}_{n}=\log _{2}\left(1+\frac{\beta_{n}\left(\alpha_{n} M+1\right)}{\frac{1}{\eta} \sum_{i=1}^{K} \beta_{i}+\frac{\alpha_{n} \beta_{n}}{\eta}-\left(1+\alpha_{n}\right) \beta_{n}+\frac{1+\sigma^{2}}{\eta \rho_{\mathrm{u}}|\chi|^{2}}}\right) .
$$

Note that in the denominator of (20), the impacts of lowresolution ADCs and RF impairments mainly occur in the term $\frac{1+\sigma^{2}}{\eta p_{u} \mid \chi^{2}}$, which unveils the compensations between resolution of ADCs and RF impairments. Increasing $\eta$ and decreasing $|\chi|$ (alternatively, increasing $\eta$ and increasing $\sigma$ ) could keep the term $\frac{1+\sigma^{2}}{\eta p_{u}|x|^{2}}$ unchanged, and vice versa. This means the uplink rate performance degradation caused by severe RF impairments could be compensated by improving the resolution of ADCs, and vice versa.

\section{Numerical Results}

In this simulation, we consider a cell with radius of 900 meters, where the $K$ users are randomly and uniformly distributed excepting a central circle of the BS with radius $r_{h}$. The geometric attenuation and shadow fading are defined as $\beta_{k}=z_{k} /\left(r_{k} / r_{h}\right)^{v}$, where $z_{k}$ is a log-normal variable with $10 \log _{10}\left(z_{k}\right) \sim \mathcal{N}\left(0, \sigma_{\text {shadow }}^{2}\right)$ [1], and $r_{k}$ is the distance between the $k$ th user and the BS. We define the uplink sum rate of the entire system as $R=\sum_{n=1}^{K} R_{n}$. The simulation parameters are listed in Table II.

Fig. 2 shows MSE of the channel estimator versus SNR with different levels of hardware impairments. We can see that coarse ADCs and hardware impairments create a floor on MSE. As opposed to the case of ideal hardware, an nonzero estimation error floor arises due to hardware impairments and 


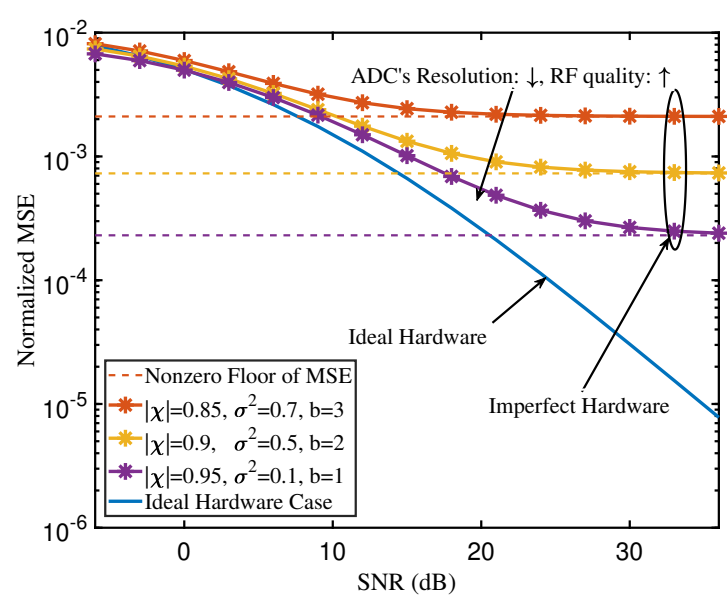

Fig. 2. MSE of channel estimator versus SNR.

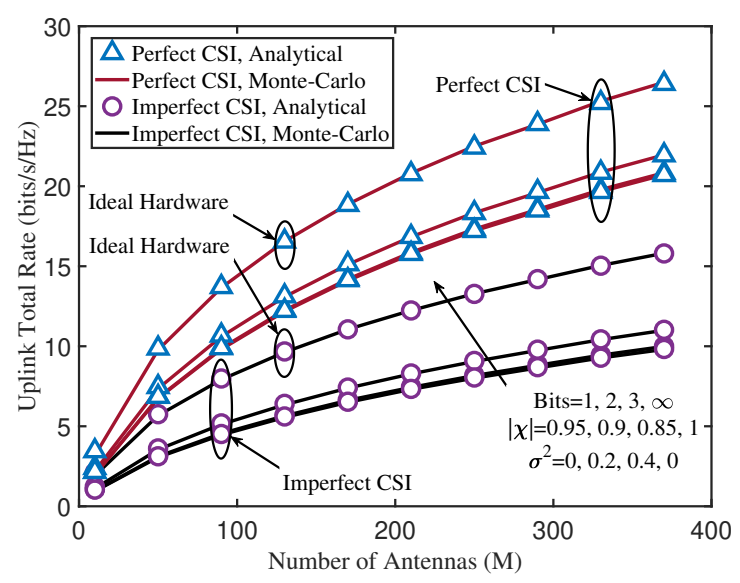

Fig. 3. Comparison between simulated result and analytical result.

TABLE II

Values of Parameters for Simulation

\begin{tabular}{|c|c|c|c|c|c|}
\hline parameters & $r_{h}$ (meters) & $\sigma_{\text {shadow }}^{2}(\mathrm{~dB})$ & $v$ & $N$ & $p_{u}(\mathrm{~dB})$ \\
\hline value & 100 & 8 & 3.8 & 10 & 10 \\
\hline
\end{tabular}

cannot be eliminated by increasing SNR, which is discussed in Remark 1.

Fig. 3 shows the approximate result in Theorem 2 and the ergodic rate in (17) versus $M$. Since the errors between the Monte-Carlo simulation of (17) and the approximate analytical uplink rate are negligible, the accuracy of the approximate expression in Theorem 2 is validated. Furthermore, we can see that the channel estimation errors cause notable loss of sum rate. Moreover, compared with the case of perfect hardware, low-resolution ADCs and RF impairments cause severe performance degradation.

Fig. 4 shows the uplink sum rate versus $M$. We can see that different levels of hardware impairments lead to the same sum rate, which illustrates a type of compensation between coarse $\mathrm{ADCs}$ and imperfect RF components for the performance degradation. This compensation could be described as that the uplink rate performance degradation caused by severe RF impairments (decreasing $|\chi|$ ) could be compensated by increasing the resolution of ADCs, and vice versa.

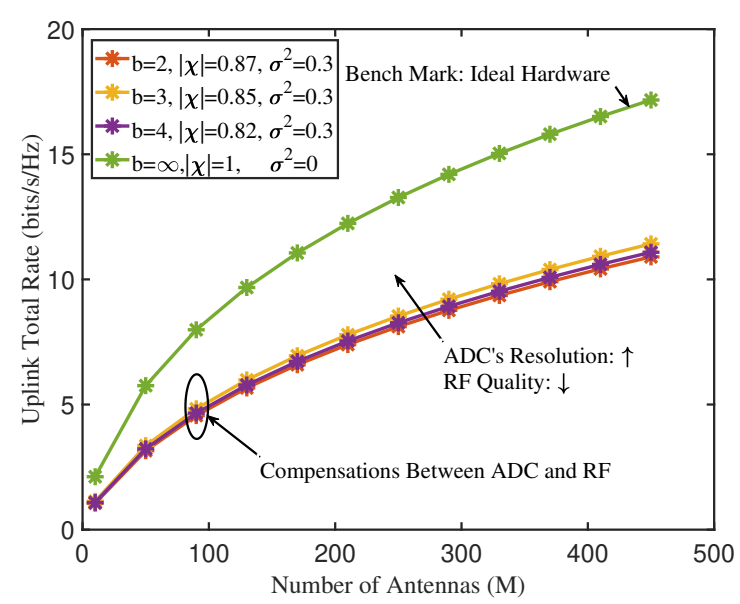

Fig. 4. Compensation between ADCs resolution and RF impairments.

\section{Conclusion}

We propose a method for channel estimation and derive a tractable expression for the uplink achievable rate of the coarsely quantized massive MIMO system with RF impairments. We show that hardware impairments and coarse ADCs create an nonzero floor on channel estimation error. Furthermore, the appreciable compensations between ADCs' resolution and RF impairments are demonstrated. These discussions support the feasibility of the deployment of coarse ADCs and imperfect RF components in massive MIMO system.

\section{REFERENCES}

[1] T. L. Marzetta, "Noncooperative cellular wireless with unlimited numbers of base station antennas," IEEE Trans. Wireless Commun., vol. 9, no. 11, pp. 3590-3600, Nov. 2010.

[2] L. Fan, S. Jin, C. K. Wen, and H. Zhang, "Uplink achievable rate for massive MIMO systems with low-resolution ADC," IEEE Commun. Lett., vol. 19, no. 12, pp. 2186-2189, Dec. 2015.

[3] J. Zhang, L. Dai, S. Sun, and Z. Wang, "On the spectral efficiency of massive MIMO systems with low-resolution ADCs," IEEE Commun. Lett., vol. 20, no. 5, pp. 842-845, May 2016.

[4] J. Xu, W. Xu, and F. Gong, "On performance of quantized transceiver in multiuser massive MIMO downlinks," IEEE Wireless Commun. Lett., vol. 6, no. 5, pp. 562-565, Oct. 2017.

[5] Y. Li, C. Tao, G. Seco-Granados, A. Mezghani, A. L. Swindlehurst, and L. Liu, "Channel estimation and performance analysis of one-bit massive mimo systems," IEEE Trans. Signal Process., vol. 65, no. 15, pp. 4075-4089, Aug. 2017.

[6] D. Tandur and M. Moonen, "Joint adaptive compensation of transmitter and receiver iq imbalance under carrier frequency offset in ofdm-based systems," IEEE Trans. Signal Process., vol. 55, no. 11, pp. 5246-5252, Nov. 2007.

[7] T. Schenk, RF Imperfections in High-rate Wireless Systems: Impact and Digital Compensation. Springer Netherlands, 2008.

[8] E. Björnson, J. Hoydis, M. Kountouris, and M. Debbah, "Massive MIMO systems with non-ideal hardware: Energy efficiency, estimation, and capacity limits," IEEE Trans. Inf. Theory, vol. 60, no. 11, pp. 71127139, Nov. 2014.

[9] A. Mezghani and J. A. Nossek, "Capacity lower bound of MIMO channels with output quantization and correlated noise," in Proc. IEEE Int. Symp. Inf. Theory, 2012.

[10] S. M. Kay, Fundamentals of statistical signal processing, Volume I: estimation theory. Prentice Hall, 1993.

[11] L. Xu, X. Lu, S. Jin, F. Gao, and Y. Zhu, "On the uplink achievable rate of massive MIMO system with low-resolution ADC and RF impairments," arXiv, 2018. [Online]. Available: http://arxiv.org/abs/1901.07893 